\section{Bürgerdialoge zwischen Technikkommunikation und Reflexion}

\author{
von Julia Hahn, Torsten Fleischer und Michael \\ Decker, Institut für Technikfolgenabschätzung \\ und Systemanalyse (ITAS), Karlsruhe
}

\begin{abstract}
In welchem Verhältnis stehen Wissenschaft, Politik und Öffentlichkeit? In diesem Text soll die Rolle von Beteiligung in Technikkommunikation am Beispiel eines großformatigen Beteiligungsprozesses näher betrachtet werden. Es wird beschrieben, in welchem Kontext ein solcher Dialog, getragen vom Bundesministeriums für Bildung und Forschung (BMBF), entstehen konnte und welche Spannungen während des Prozesses aufgetreten sind. Darüber hinaus werden einige Schlussfolgerungen für Technikkommunikation gezogen, die für zukünftige Beteiligungsbemühungen wichtig sind. Dies scheint vor dem Hintergrund der zunehmenden Forderung nach Mitbestimmung besonders relevant.
\end{abstract}

What is the relationship between science, politics and society? In the following text we discuss the role of participation in technology communication by looking at the example of a large-scale engagement process. We describe in which context such a dialogue, initiated and shaped by the German Ministry of Education and Research, could develop and which tensions occurred. Further, we make several conclusions regarding technology communication that can be helpful for future engagement ambitions. This seems especially important due to rising demands for more participation.

\section{Vorbemerkung}

Versucht man, sich dem Phänomen Technikkommunikation zu nähern, gerät man schnell in konzeptionell und terminologisch kompliziertes Terrain. Bekannt ist, dass Wissenschaftskommunikation seit einiger Zeit sowohl wissenschaftlicher Gegenstand ist als auch in der Öffentlichkeit kontrovers diskutiert wird. Pansegrau et al. haben schon 2011 - leicht euphemistisch - darauf hingewiesen, dass Wissenschaftskommunikation ein „etwas doppeldeutiger" Begriff sei, der zum einen sowohl die Kommunikation „der" Wissenschaft als auch die Kommunikation ,über sie“ mit einer „unspezifizierten aber möglichst breiten" Öffentlichkeit beschreibe, und der zum anderen auch die Kommunikation der Wissenschaft über die Massenmedien sowie der Massenmedien über die Wissenschaft in den Blick nehme. Folgt man dieser Beschreibung, wäre Technikkommunikation also die Kommunikation „der" Technik wie auch Kommunikation „über“" Technik, sowohl direkt zwischen Akteuren und der allgemeinen Öffentlichkeit als auch vermittelt durch Massenmedien? Wer genau aber wäre „die Technik“ und ihre Akteure? Anders als „die Wissenschaft“ beschreibt „die Technik“ eben kein einigermaßen sauber $\mathrm{zu}$ fassendes soziales Teilsystem. Und befasst sich der Großteil von Wissenschaftskommunikation nicht vorzugsweise mit Erwartungen und Visionen hinsichtlich der Nutzbarmachung und Verwendung ihrer Ergebnisse, ist also genau genommen sowieso eher Technikkommunikation? Würde eine solche Trennung für moderne Technowissenschaften überhaupt sinnvoll aufrechterhalten werden können?

Dieser Aufsatz kann die angerissene Diskussion nicht führen. Es geht hier vielmehr darum, die Rolle von Bürgerdialogen in der (Wissenschafts- und) Technikkommunikation genauer zu eruieren. Wir wollen aber eingangs festhalten, dass wir beide Begriffe in der Folge eher synonym verwenden werden, auch in der Bezugnahme auf Resultate der Forschung zur Wissenschaftskommunikation. Dies ist zum einen der - hier unbewiesen bleibenden - Annahme geschuldet, dass zumindest bei Kommunikation „über etwas“" Wissenschaft und Technik sowieso nicht mehr sauber trennbar sind, zum anderen bezieht sich unser Fallbeispiel auf die Bürgerdialoge Zukunftstechnologien des BMBF, das sich selbst sowohl der Förderung wissenschaftlicher Forschung als auch von Innovationsprozessen verpflichtet sieht.

\section{2 „Let's talk about..." - Wie interagieren Wissenschaft, Politik und die Bürger?}

In den letzten Jahren kann zunehmend von einem ,participatory turn“ (Jasanoff 2003, S. 235) gesprochen werden. Schlagwörter wie Bürgerbeteiligung, Citizen Science, Open Innovation oder Responsible Research and Innovation formen 
immer mehr die Interaktionen zwischen Wissenschaft und Gesellschaft und finden sich vermehrt in Ausschreibungen der EU oder der Bundesregierung wieder, wenn es um forschungspolitische Rahmungen geht. Jedoch ist es oft unklar, wie diese Interaktionen stattfinden sollen. Mit welchem Ziel werden Bürger zu einem „Nano-Dialog“ eingeladen? Woran wollen Akteure eigentlich partizipieren, wenn sie sich an Citizen-Science-Projekte beteiligen? Wie lässt sich „engagement“ operationalisieren und in verschiedene Innovationsphasen integrieren? Geht es darum, dass „die Öffentlichkeit" ein besseres Verständnis von „der Wissenschaft" bekommt, oder dass sich Wissenschaft ein besseres Gespür für Bürgermeinungen verschafft?

Eines scheint sicher: Das „deficit model“ (Durant 1999), das davon ausgeht, dass Bürgern nur mehr Experteninformationen fehlen, um Technologien zu akzeptieren, ist überholt. Heute finden Interaktionen auf vielen Ebenen zwischen unterschiedlichen Akteuren in wechselnden Rollen statt. Diese sind spezifisch und kontextuell. In diesem Zusammenhang kann es nützlich sein, ein konkretes Beispiel einer solchen Interaktion näher zu beleuchten, um Knackpunkte oder Spannungen aufzuzeigen, die bei Beteiligungs- prozessen eine wichtige, in der Diskussion darüber aber oft vernachlässigte Rolle spielen. Der Bürgerdialog Zukunftstechnologien ist hier interessant, da es ein Beteiligungsprojekt war, bei dem ein politischer Akteur (Bundesministerium für Bildung und Forschung, BMBF) explizit mit Bürgern in den Austausch ging, um einerseits über neue technologische Entwicklungen $\mathrm{zu}$ informieren, aber auch, um Rückmeldungen zu Forschungsausrichtungen und -schwerpunkten zu bekommen.

\section{Ein Bürgerdialog über Zukunft}

Das Projekt „Bürgerdialog Zukunftstechnologien" das von 2011 bis 2013 stattfand, war der erste großformatige, länger laufende nationale forschungspolitische Beteiligungsprozess, der von einer politischen Institution, dem BMBF, initiiert, mit entworfen und aktiv begleitet wurde. Die Themen des Bürgerdialogs waren Energietechnologien für die Zukunft, Hightech-Medizin und demografischer Wandel.

Der Aufbau des Dialogkonzepts folgte einem Phasenmodell (Abb. 1), das aber je nach

Abb. 1: Struktur des Bürgerdialogs Zukunftstechnologien

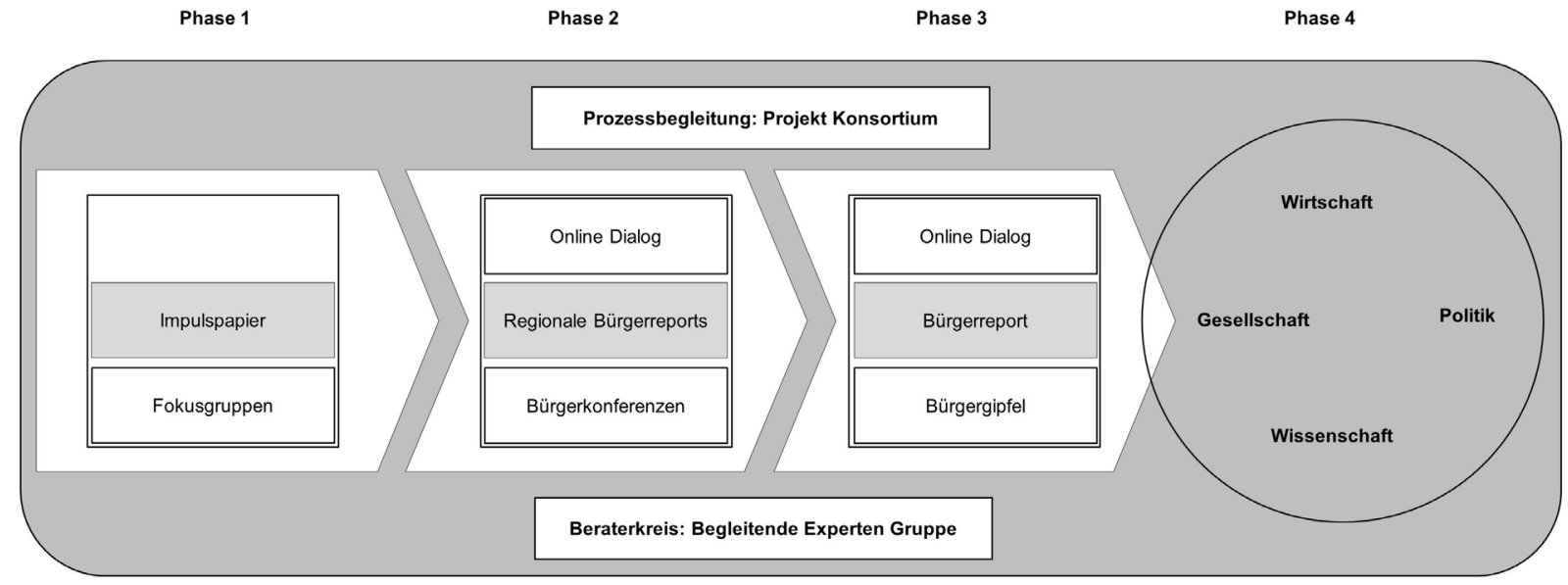

Phase 1: Identifikation von wichtigen Themen und erste Reflexionen in Fokusgruppen $\rightarrow$ Impulspapier Phase 2: Bürger diskutierten Hoffnungen und Bedenken und erste Handlungsempfehlungen bei den regionalen Bürgerkonferenzen $\rightarrow$ regionale Bürgerreports

Phase 3: Bürger entwickelten konkrete Empfehlungen beim Bürgergipfel

$\rightarrow$ Bürgerreport

Phase 4: Die Ergebnisse des Bürgerdialogs (v. a. Bürgerreport) wurden an Vertreter aus Wirtschaft, Gesellschaft, Politik und Wissenschaft überreicht.

Quelle: Eigene Darstellung 
Thema leicht unterschiedlich umgesetzt wur$\mathrm{de}^{1}$. In einer ersten, eher explorativen Phase wurden Fokusgruppen organisiert (beim ersten Dialogthema Energie gab es diese aus Zeitgründen nicht, weil das BMBF sehr schnell nach dem Verkünden der Energiewende in den Dialog treten wollte) um Rahmungen, thematische Schwerpunkte oder auch kontroverse Positionen frühzeitig zu identifizieren. Deren Auswertung ist dann in die Erstellung eines Impulspapiers geflossen, das vom Projektkonsortium verfasst und nach Abstimmung mit dem BMBF an die eingeladenen Bürger der regionalen Bürgerkonferenzen im Vorfeld verteilt wurde. Diese Bürgerkonferenzen fanden mit jeweils ca. 100 repräsentativ ausgewählten Bürgern in verschiedenen Regionen Deutschlands statt. Pro Thema gab es sechs bis acht solcher Konferenzen, deren Ergebnisse in regionalen Bürgerreports festgehalten wurden. Somit konnten insgesamt zwischen 600 bis 800 Bürger in ganz Deutschland zu jedem Thema diskutieren und regionale Bürgerreports verfassen.

Die regionalen Reports waren dann die Basis für den Bürgergipfel, der für jedes der Themen Energie, Hightech Medizin und demografischer Wandel in Berlin im Paul-Löbe-Haus des Deutschen Bundestags stattfand. Hierzu wurden jeweils ca. 100 Bürger, die auch schon an den regionalen Bürgerkonferenzen teilgenommen hatten, eingeladen, um zwei Tage über die bisherigen Ergebnisse zu diskutieren und Handlungsempfehlungen für einen abschließenden Bürgerreport zu verfassen. Dieser wurde dann an Vertreter aus Politik (z. B. an die damalige Ministerin Annette Schavan), Gesellschaft, Wissenschaft und Wirtschaft übergeben. Während dieser Zeit lief parallel ein Onlinedialog und es gab Rückmeldungen zu den Ergebnissen von einem Beraterkreis, zusammengesetzt aus Wissenschaftlern und Vertretern aus Gesellschaft und Wirtschaft.

\section{4 „Politikberatung durch Bürger“ - Ziele und Spannungen der Bürgerdialoge}

Die Bürgerdialoge sind in einer bestimmten politischen Atmosphäre entstanden. Die Initiierung eines solchen Vorhabens bedarf eines expliziten politischen Willens hin zu einer Öffnung und Einbeziehung der Öffentlichkeit. Im Koalitions- vertrag von CDU/CSU und FDP aus dem Jahr 2009 finden sich Aussagen, die auf große Erwartungen an Beteiligungsverfahren in Bezug auf Konfliktlösung und Akzeptanzförderung deuten. Hier heißt es u. a.:

„Deshalb geht es uns darum, dass in Deutschland, dem Land der Ideen, neue Technologien nicht nur entwickelt, sondern auch angewandt werden. Dazu brauchen wir auch einen umfassenden Dialog über Zukunftstechnologien mit und unter den Bürgerinnen und Bürgern. Wir stehen für eine zukunftsorientierte Kultur der Chancen. Wir wollen wieder eine optimistische und technik- und innovationsfreundliche Gesellschaft werden." (Koalitionsvertrag 2009, S. 63)

Es zeigt sich, dass Handlungsbedarf bei der Interaktion zwischen Wissenschaft, Innovationsakteuren und der Öffentlichkeit gesehen wird. Aus der - in ihrem Zustandekommen unklaren - Wahrnehmung einer eher ablehnenden Haltung gegenüber neuer Technologie und Innovation wird auf die Notwendigkeit der Entwicklung einer gegenüber Technik und Innovation offenen und sogar optimistischen Kultur geschlossen. Im Koalitionsvertrag von 2013 von CDU/CSU und SPD wird dies nochmals aufgegriffen:
„Wir wollen Bürgerinnen und Bürger und die Akteure der Zivilgesellschaft konsequent in die Diskussion um Zukunftsprojekte und die Ausgestaltung von Forschungsagenden ein- binden. Wir wollen neue Formen der Bürger- beteiligung und der Wissenschaftskommuni- kation entwickeln und in einem Gesamtkon- zept zusammenführen." (Koalitionsvertrag 2013, S. 106)

Hier wird beschrieben, wie eine solche „Kultur der Chancen" ausgestaltet werden soll. Beteiligungsverfahren und Wissenschaftskommunikation sind hierbei Mittel, um die Interaktionen zwischen Wissenschaft, Politik und Gesellschaft auszugestalten und zu fördern. Eines der Verfahren, diesen Austausch zu fördern, waren die oben beschriebenen Bürgerdialoge Zukunftstechnologien.

In einer im Sommer 2010 veröffentlichten Ausschreibung für einen Dienstleistungsauftrag zur Konzeption und Durchführung von Bürgerdialogen hatte das BMBF seine Vorstellungen 
konkretisiert. Im Ausschreibungstext finden sich einige Zielbeschreibungen, die wichtig sind in Bezug auf die Erwartungen an einen solchen Dialog. Beispielsweise ginge es darum, einen „kontinuierlichen Diskurs mit der Gesellschaft über kontrovers diskutierte Zukunftstechnologien zu führen, um deren Akzeptanz zu erhöhen“ (BMBF 2010, S. 2). Die Dialoge sollten Bürger breit einbeziehen und ihnen die Möglichkeit geben, sich ,über Schlüsseltechnologien und Forschungsvorhaben $\mathrm{zu}$ informieren, [... und] die Ergebnisse dieses Meinungsbildungsprozesses gegenüber Politik, Wirtschaft und Wissenschaft zu vertreten“ (BMBF 2010, S. 4). Als „Vermittlungselement Verständigung über Konflikte bei forschungspolitischen Themen" (BMBF 2010, S. 2) sollten die Dialoge eine Möglichkeit sein, über Ausrichtung und Entwicklungen von Forschung zu informieren, also ein Informationsbzw. Kommunikationsmittel sein. Hierzu zählt auch eine Erarbeitung eines „Gesamtkonzepts zur Öffentlichkeitsarbeit des Bürgerdialogs insgesamt, d. h. die Etablierung einer ,Marke' und deren fortlaufende kommunikative Vermittlung in eine breite Öffentlichkeit" (BMBF 2010, S. 5). Somit war der Anspruch an die Dialoge nicht nur, repräsentativ ausgewählte Bürger direkt einzubeziehen, sondern auch ein Instrument zu entwickeln, das mit „der Öffentlichkeit“ im weiteren Sinne kommunizieren kann.

Ein anderer Anspruch an die Entwicklung eines Dialogprozesses war die Möglichkeit zur Reflexion. Das BMBF wollte einen Raum schaffen, um ,auch eine Rückmeldung zu den geplanten Innovationen im kritischen Dialog mit Bürgerinnen und Bürgern zu erhalten" (BMBF 2010, S. 2). Hierbei sollte der Dialogprozess als „Frühwarnsystem (frühzeitige sachliche Information und Diskussion) [und] als Orientierungselement (konkrete Vorschläge, Ideen, Ansprüche der Bürgerinnen und Bürger aufnehmen und in den politischen Prozess einbringen)" dienen (BMBF 2010, S. 2). Die Bürger sollten breit einbezogen werden, und es sollte die Möglichkeit gegeben werden, ,sich in einem öffentlichen Diskurs eine Meinung zu bilden“ (BMBF 2010, S. 2). In der Zusammenschau dieser beiden Begründungslinien scheint also die Intention des Initiators zu sein, die Dialoge von Anfang an in einer Dop- pelrolle zu etablieren: Zum einen als Element (oder Instrument?) der Wissenschafts- und Technikkommunikation des Forschungsministeriums, zum anderen als Möglichkeit der Reflexion über Technologiefragen durch Bürger in einem deliberativen Prozess unter Einbezug von Experten aus Wissenschaft, Wirtschaft, Forschungspolitik und -administration.

Es muss hier offen bleiben, inwieweit den Autoren der Ausschreibung die Spannung zwischen diesen beiden Zielsetzungen bewusst war. In der Durchführung der Dialoge zeigte sie sich deutlich. So wurde in der teilnehmenden Beobachtung der Dialoge immer wieder festgestellt, dass als „Wissensressource“ teilnehmende Experten, sobald sie eine aus Sicht der Bürgerinnen und Bürger zu interessengeleitete, bestimmte Techniken promovierende Perspektive einnahmen, sehr kritisch befragt und in einigen Fällen sogar (höflich, aber bestimmt) aus den Dialogrunden verabschiedet wurden.

Als ein weiteres Indiz für diese Spannung kann die Neurahmung der Themen während der Dialoge verstanden werden. Im ersten Dialog zum Thema Energie war die ursprünglich von Initiator vorgenommene Rahmung sehr an Energietechnologien und den damit verbundenen deutschen Forschungsstärken orientiert. Im Mittelpunkt standen beispielsweise Energieeffizienz, erneuerbare Energie, Energienetze sowie „Brückentechnologien“, also Übergangstechnologien, die bis zur Realisierung der Energiewende benötigt würden. Im weiteren Prozess wurde dann die Diskussion durch die Bürger neu gerahmt und $\mathrm{u}$. a. Dezentralisierung von Energieproduktion, politische Steuerung, Forschung und Entwicklung, Erziehung, Bildung, Information und Beratung sowie der Bürger als Mitgestalter in den Fokus gerückt. Hierbei ging der Blick eher zu gesamtgesellschaftlichen Aspekten der Energie der Zukunft. Beim Dialog zur Hightech-Medizin war die ursprüngliche Rahmung ebenfalls an konkreten Technologien orientiert. So wurde über neuronale Implantate, Telemedizin und Intensiv- und Palliativmedizin diskutiert. Im Prozess der Dialoge fand auch hier eine Neurahmung statt, die dann die Diskussion bei dem Bürgergipfel bestimmte. Hierbei wurde das Augenmerk auf Datenschutz, informier- 
te Selbstbestimmung, Zugangsgerechtigkeit zu neuen Technologien und menschliche Betreuung und Rolle der Technik gelegt. Hier zeigte sich eine Verschiebung in Richtung eher individueller Bedürfnisse von Patienten oder Betroffenen.

Bei beiden Themenbereichen ging der Fokus von der Technologie oder konkreten Innovation hin zu deren breiteren gesellschaftlichen Auswirkungen oder einer individuellen bzw. Betroffenenperspektive. Beim Thema demografischer Wandel war die Neurahmung nicht so stark, da schon von Anfang an durch Themenbereiche wie Zusammenleben, lebenslanges Lernen und Arbeitswelt eher gesellschaftliche Aspekte die ursprüngliche Rahmung bestimmten. Aber auch diese Bereiche wurden während des Prozesses noch konkretisiert, z. B. Förderung des Austauschs zwischen Jung und Alt, Gestaltung einer befähigenden Infrastruktur, Bildungswesen neu gestalten oder ältere Arbeitnehmer in der Arbeitswelt.

Das BMBF hatte sich während der Dialoge auf diese neuen Rahmungen eingelassen und auf die Reflexion der Bürger reagiert. Wie dies im Ministerium aufgenommen wurde, wird von Christoph Braß, damaliger Leiter der Unterabteilung „Bildungs- und forschungspolitische Grundsatzfragen" und einer der Hauptakteure der Bürgerdialoge, wie folgt beschrieben:

„Ja natürlich, man ist ja überrascht, wenn man merkt, dass sich die Geschäftsgrundlage verändert bzw. dass den Bürgern andere Aspekte oder auch andere Themenbereiche wichtiger sind als diejenigen, mit denen man selber das Gespräch gestartet hat. (...) So kamen bei den Dialogen zur Hightech-Medizin die Menschen sehr rasch auf eine grundlegende ethische Debatte. Das war für uns ein klares Signal, dass es da offenbar noch Desiderate gibt, und ein Bedürfnis darüber $\mathrm{zu}$ diskutieren und jetzt noch nicht über die Frage zu diskutieren, welche Maschinen sozusagen sinnvoll und weniger sinnvoll sind. Das ist ja eine ernstzunehmende Rückmeldung, die wir dann ja auch entsprechend aufgegriffen haben, auch wenn sie in der Konsequenz bedeutet hat, dass wir am Ende vielleicht nicht auf alle Fragen, die uns wichtig gewesen wären, schon eine Antwort erhalten hätten. Dafür sind wir von den Bürgern aber mit anderen Fragen konfrontiert worden, die ihnen wichtig sind und die wir eben noch nicht in dem Maße im Blick hatten. Von daher hat der Dialog da schon auf beiden Seiten zu einem Erkenntnisgewinn geführt.“ (Decker/Fleischer 2014, S. 59)

Hier zeigt sich, dass sich während der Laufzeit der Dialoge die Erwartungen insofern verschoben hatten, dass es immer weniger um die Kommunikationselemente ging und stattdessen die Reflexion in Form der Neurahmung im Mittelpunkt stand. Die Bürgerdialoge wurden also mehr und mehr „dialogisch“. Hierbei spielt auch der Umgang mit dieser Situation innerhalb eines Beteiligungsverfahrens eine wesentliche Rolle. Der Bürgerdialog war offen genug, um auf diese Reflexion zu reagieren und eine Neurahmung zuzulassen. Gleichzeitig war es wichtig, einen Weg zu finden, wie mit den Erwartungen seitens der Bürger umzugehen war. Christoph Braß stellt hierzu klar:

\section{„Sie muss zum einen Wertschätzung und Dankbarkeit vermitteln gegenüber den Bür- gerinnen und Bürgern und das klare Signal: ,Wir nehmen ernst, was ihr uns sagt'. Zugleich darf sie aber auch nicht unehrlich werden und mehr versprechen als wir halten können. In- sofern war auch bei diesen Erwiderungen der Ministerin oder des Staatssekretärs die oberste Prämisse, deutlich zu machen: Es ist uns wich- tig, was ihr hier liefert, wir nehmen das ernst, aber wir wollen auch nichts versprechen, was wir nicht einlösen können." (Decker/Fleischer 2014, S. 60)}

Für Beteiligungsverfahren, besonders für solche in einem politischen Kontext wie die Bürgerdialoge, scheint es wichtig, diese flexibel genug zu gestalten, dass Anpassungen und Änderungen auch während des Prozesses möglich sind. Aber auch ein ,expectation management" in Sinne der Klarstellung, was von den verschiedenen Seiten erwartet werden kann, scheint entscheidend.

\section{Von „Public Understanding of Science“" zu „A Better Understanding of the Public“}

Wie oben beschrieben, waren die „Bürgerdialoge Zukunftstechnologien“ durch verschiedene Ziele geprägt. Diese schwankten zwischen „Politikberatung durch Bürger", also dem Erarbeiten von Empfehlungen für Politik, Wirtschaft, Wissen- 
schaft und Gesellschaft im Zuge von deliberativen Verfahren, und Informationszielen (Sichtbarkeit des Ministeriums und seiner Programme sowie der öffentlich geförderten Wissenschaft als Ganzes). Außerdem schien es, auch eine technologiepolitische Erwartung seitens des Ministeriums an die Dialoge zu geben, da Akzeptanz für neue Technologien und Innovation geschaffen werden sollte. Diese Ziele schließen sich einerseits nicht aus, stehen aber andererseits teilweise in einem Spannungsverhältnis zueinander.

Diese, beim Eintreten in den Dialogprozess nicht hinreichend reflektierte Spannung, hatte Rückwirkungen in seine inhaltliche Gestaltung hinein. Erst durch die inhaltliche Neurahmun einzelner Themenschwerpunkte durch die Bürger während der Dialoge, wurden die Themen „verhandlungsfähig“. Die Bürger haben in einer komplexen Themenumgebung neue, eigene Schwerpunkte gesetzt und Aspekte hervorgehoben, die für sie selbst wichtig waren, und sich von den ursprünglichen, forschungspolitisch vorgenommenen Rahmungen mit zumindest partieller technikkommunikativer Absicht unterschieden haben. Somit fand ein wirklicher Dialog erst nach dessen Rekonfiguration statt: Die Bürger hatten die Ebene des reinen „Empfängers" von Information verlassen und sich auf Rückmeldung und Reflexion konzentriert.

Bemerkenswert ist, dass sich das BMBF als Initiator auf diese Rekonfiguration eingelassen hatte und somit eine Erweiterung der Zielsetzungen um die der Herstellung eines kollektiven Lernprozesses für alle Beteiligten zuließ. Aus Sicht des BMBF gibt Christoph Braß einige Einschätzungen:

„Und schließlich als zentrales Anliegen des BMBF: Der Wunsch, einen geschützten Raum zu schaffen, wo Politik, Wissenschaft und Bürger sich in einem Klima der gegenseitigen Wertschätzung begegnen können - ohne die Notwendigkeit, Fensterreden zu halten, ohne die (gefühlte) Notwendigkeit, eigene Schwächen nicht eingestehen zu können. [...] Ich glaube, wir haben als Haus noch einmal auf eine neue Weise gelernt, zuzuhören und jenseits der Fachdiskurse, die wir täglich führen, die Alltagsvernunft der Bürgerinnen und Bürger wahrzunehmen.“(Decker/Fleischer 2014, S.57)
Aber auch die Bürger mussten sich zunächst in ihrer Rolle als gefragte Berater finden, konnten dies aber durch die beschriebenen Neurahmungen schnell für sich ausgestalten. Dies wiederum war nur möglich, weil das Dialogformat und die beteiligten Institutionen hinreichend flexibel waren, dies „live“ umzusetzen. Für die involvierten Wissenschaftler und Wissenschaftsadministratoren beinhalteten die Dialoge und ihre Ergebnisse eine Selbstreflexion über die eigene Rolle - eine Situation, mit der sich einige Gäste aus der Wissenschaft erkennbar schwer taten. Zwar liegt hierzu nur anekdotische Evidenz vor, diese weist aber darauf hin, dass die beteiligten Bürger den Experten zwar eine sehr wichtige Rolle als Informationsquelle im Dialogprozess einräumten, ihnen aber zugleich allein nicht die faktische Entscheidung über Themen und Technologien überlassen wollten. Eine Reihe von Experten bestand andererseits in den Dialogrunden darauf, dass Entscheidungen über technologische $\mathrm{Zu}$ künfte anhand von „objektiven Fakten“ getroffen werden sollten, die nun einmal von Experten am besten verstanden würden. Kaum weniger problematisch war, dass sich in mehreren Fällen Experten in Bürgerdiskussionen einmischten, lange monologisierten oder versuchten, ihnen wichtige Themen zu befördern oder rhetorisch „durchzusetzen“.

Festzuhalten ist auch, dass die kommunikativen Effekte der Bürgerdialoge in Bezug auf die Öffentlichkeit hinter den Erwartungen weit zurück blieben. Das Medienecho war - trotz intensiver Bemühungen einer eigens engagierten Medienagentur - vergleichsweise gering. Das Verfahren der Bürgerdialoge selbst ist anscheinend keine Nachricht mehr, auch lautstark ausgetragene Konflikte, politische Instrumentalisierungen oder Skandalisierungen blieben aus, so dass überregionale Medien kaum berichteten. Eine weitere - allerdings unbelegte - Vermutung lautet, dass ein „Bürgerdialog Zukunftstechnologien" auch schlicht nicht in die Organisationsstrukturen von großen Medienorganisationen passt - kurz gesagt: nicht politisch genug für das Politikressort, nicht wissenschaftsnah genug für das Wissenschaftsressort ist. Regionale Medien berichteten dann doch in merklichem Umfang über die regionalen Bürgerkonferenzen zum de- 
mografischen Wandel, da sich Lokalpolitiker in größerer Zahl an ihnen beteiligten und die dort geführten Diskussionen unmittelbar anschlussfähig zu lokalen Politikprozessen waren.

Das früh bemerkte geringe Medienecho führte in der parallel geführten Prozessreflexion zwischen Initiator und beteiligten Agenturen und Forschungseinrichtungen immer wieder $\mathrm{zu}$ Diskussionen, ob man denn die Dialogprozesse nicht thematisch und prozedural stärker an einem - vermuteten - Medieninteresse ausrichten sollte, um ihre kommunikative Rolle zu stärken. Die Positionen hierzu waren vor allem anfangs durchaus konträr, letztlich wurde dann aber doch beschlossen, von einem solchen Vorgehen Abstand zu nehmen. Ein wichtiger Grund dafür war, dass eine - aus unserer Sicht sehr berechtigt befürchtete - Wahrnehmung der Dialoge als eine primär durch PR-Interessen geprägte Veranstaltung sowohl deren Ergebnisse entwertet als auch das Format als Ganzes delegitimiert hätten. Wissenschafts- und Technikkommunikation kann mithin bestenfalls ein Nebenziel von Bürgerdialogverfahren sein - und das auch eher im Sinne des Aufmerksammachens auf neue Themen und ihre sozialen Implikationen statt als Instrument zur Kommunikation von Inhalten wissenschaftlicher und technischer Entwicklungen. Zwar ist die Kenntnis des Zweitgenannten Voraussetzung für das erstere, diese Inhaltskommunikation sollte aber bereits im Vorfeld der Reflexion und auch formal losgelöst vom Dialogverfahren stattfinden. Eine solche zeitliche Abstufung und eine klarere Zielpriorisierung sollten in zukünftige Dialogverfahren stärker Eingang finden.

\section{Anmerkung}

1) Für eine detaillierte Beschreibung der Dialoge und deren Entstehung s. Decker/Fleischer 2014; Hahn et al. 2014.

\section{Literatur}

BMBF - Bundesministerium für Bildung und Forschung, 2010: Ausschreibung für einen Dienstleistungsauftrag. Konzeption und Durchführung von Bürgerdialogen des Bundesministeriums für Bildung und Forschung sowie deren Begleitung durch Öffentlichkeitsarbeit (1.4.2010)
Decker, M.; Fleischer, T., 2014: Bürgerdialoge: Die Notwendigkeit für solche Formate ist auf jeden Fall gegeben. Interview mit Christoph Braß über die Bürgerdialoge ,Zukunftstechnologien und Zukunftsthemen" des BMBF. In: Technikfolgenabschätzung - Theorie und Praxis 23/2 (2014), S. 56-62

Durant, J., 1999: Participatory Technology Assessment and the Democratic Model of the Public Understanding of Science. In: Science and Public Policy 26/5 (1999), S. 313-319

Hahn, J.; Seitz, St.B.; Weinberger, N., 2014: What Can TA Learn from ,the People"? A Case Study of the German Citizens' Dialogues on Future Technologies. In: Michalek, T.C.; Hebakova, L.; Hennen, L. et al. (Hg.): Technology Assessment and Policy Areas of Great Transitions. Prag, S. 165-170

Jasanoff, S., 2003: Technologies of Humility: Citizen Participation in Governing Science. In: Minerva 41 (2003), S. 223-244

Koalitionsvertrag, 2009: Wachstum. Bildung. Zusammenhalt. Koalitionsvertrag zwischen CDU, CSU und FDP, 17. Legislaturperiode

Koalitionsvertrag, 2013: Deutschlands Zukunft gestalten. Koalitionsvertrag zwischen CDU, CSU und SPD, 18. Legislaturperiode

Pansegrau, P.; Taubert, N.; Weingart, P., 2011: Wissenschaftskommunikation in Deutschland. Ergebnisse einer Onlinebefragung. Eine Untersuchung im Auftrag des Deutschen Fachjournalisten-Verbandes (DFJV). Berlin

\section{Kontakt}

Julia Hahn

Institut für Technikfolgenabschätzung und

Systemanalyse (ITAS)

Karlsruher Institut für Technologie (KIT)

Karlstraße 11, 76133 Karlsruhe

Tel.: +49 721 608-28024

E-Mail: julia.hahn@kit.edu 